\title{
Development of bio-yoghurt chewable tablet: a review
}

\begin{abstract}
Purpose - This paper aims to discuss the limitations surrounding the yoghurt industry and challenges to producing a bio-yoghurt tablet. The paper looks into challenge facing the yoghurt industry, such as manufacturing and distribution, its short shelf life, heat-sensitivity and relatively heavy weight. It further looks into the selection of strains, excipients and storage conditions with special consideration towards maintaining the viability of the probiotics inside bio-yoghurt tablets. The paper also discusses yoghurt standards and definitions across various countries and suggests a more uniform standard be embraced across countries for ease of categorization and production.

Design/methodology/approach - The paper is divided into a few major sections; each exploring various aspects of the yoghurt industry. Topics discussed include challenges in yoghurt production and storage; processes involved in bio-yoghurt tablet production, e.g. maximising viability, choice of excipients and more; market trends of yoghurt consumption and potential; and various food standards in countries around the world with a focus on yoghurt.
\end{abstract}

Findings - The review finds that yoghurt is a segment of the food industry with big growth potential. Most of the problems associated with yoghurt, i.e. poor shelf life, and heavy weight, can be circumvented by transforming it into a bio-yoghurt tablet. The paper further identifies food standard variations among different countries around the world which could impede yoghurt manufacture and acceptance.

Originality/value - This paper looks the various challenges surrounding the increased uptake of yoghurt, specifically in the Asian markets and suggests a viable option to overcome this problem, i.e. the use of a bioyoghurt tablet. Should the worldwide bodies come together and agree to a universal standard involving yoghurt, the industry may see an even bigger expansion.

Keyword: Probiotic; Market trend; Food regulation; Yoghurt; Chewable tablet; Pharmacopoeia standard 